\title{
Intermittent Theta Burst Over M1 May Increase Peak Power of a Wingate Anaerobic Test and Prevent the Reduction of Voluntary Activation Measured with Transcranial Magnetic Stimulation
}

\author{
Louis-Solal Giboin*, Patrick Thumm, Raphael Bertschinger and Markus Gruber \\ Sensorimotor Performance Lab, Sport Science Department, Universität Konstanz, Konstanz, Germany
}

Despite the potential of repetitive transcranial magnetic stimulation (rTMS) to improve performances in patients suffering from motor neuronal afflictions, its effect on motor performance enhancement in healthy subjects during a specific sport task is still unknown. We hypothesized that after an intermittent theta burst (iTBS) treatment, performance during the Wingate Anaerobic Test (WAnT) will increase and supraspinal fatigue following the exercise will be lower in comparison to a control treatment. Ten subjects participated in two randomized experiments consisting of a WAnT 5 min after

OPEN ACCESS

Edited by:

Nuno Sousa

University of Minho, Portugal

Reviewed by:

Yasuyuki Ishikawa

Maebashi Institute of Technology,

Japan

Pablo Arias,

University of A Coruña, Spain

${ }^{*}$ Correspondence:

Louis-Solal Giboin

louis-solal.giboin@uni-konstanz.de

Received: 22 February 2016 Accepted: 08 July 2016 Published: 19 July 2016

Citation:

Giboin L-S, Thumm P, Bertschinger R and Gruber M (2016) Intermittent

Theta Burst Over M1 May Increase Peak Power of a Wingate Anaerobic Test and Prevent the Reduction of Voluntary Activation Measured with Transcranial Magnetic Stimulation. Front. Behav. Neurosci. 10:150. doi: 10.3389/fnbeh.2016.00150 either an iTBS or a control treatment. We determined voluntary activation (VA) of the right knee extensors with TMS (VA $\mathrm{TMS}_{\mathrm{TMS}}$ ) and with peripheral nerve stimulation $\left(\mathrm{VA}_{\mathrm{PNS}}\right)$ of the femoral nerve, before and after the WAnT. T-tests were applied to the WAnT results and a two way within subject ANOVA was applied to VA results. The iTBS treatment increased the peak power and the maximum pedalling cadence and suppressed the reduction of VA $A_{T M S}$ following the WAnT compared to the control treatment. No behavioral changes related to fatigue (mean power and fatigue index) were observed. These results indicate for the first time that iTBS could be used as a potential intervention to improve anaerobic performance in a sport specific task.

Keywords: rTMS, supraspinal fatigue, central fatigue, performance, cycling, muscle fatigue, power, iTBS

\section{INTRODUCTION}

Repetitive transcranial magnetic stimulation (rTMS) may be able to induce transient plasticity in cortical neural networks and thus can modify corticospinal excitability as well (Ziemann, 2004; Hoogendam et al., 2010). Its application over the primary motor cortex (M1) has been widely used as a therapeutic tool for many conditions with various levels of success

Abbreviations: iTBS, intermittent theta burst; MEP, motor evoked potential; MSO, maximal stimulator output; MVC, maximal voluntary contraction; PNS, peripheral nerve stimulation; rTMS, repetitive transcranial magnetic stimulation; TMS, transcranial magnetic stimulation; VL, vastus lateralis; VA, voluntary activation; $\mathrm{VA}_{\mathrm{TMS}}$, voluntary activation estimated with transcranial magnetic stimulation; VAPNS, voluntary activation estimated with peripheral nerve stimulation; WAnT, Wingate Anaerobic Test. 
(Ziemann, 2005; Ridding and Rothwell, 2007; Lefaucheur et al., 2014; Palm et al., 2014). Several rTMS protocols were applied to patients suffering from a diversity of motor neuronal disorders, which consistently improved motor performance or even led to the recovery of motor function (Lefaucheur et al., 2004; Khedr et al., 2006; Kakuda et al., 2013; Tretriluxana et al., 2013; Yang et al., 2013). In healthy subjects, applications of rTMS protocols have been tested more sparsely, and only a few studies looked at performance in sport specific tasks (Muellbacher et al., 2000; Schlaghecken et al., 2003; Carey et al., 2006; Hortobagyi et al., 2009; Ward et al., 2010; Censor and Cohen, 2011; Teo et al., 2011). In a fundamental study, Benwell et al. (2006) examined the impact of rTMS in a maximal performance task. The authors were able to demonstrate that during a continuing maximal isometric voluntary pinch grip, the rate of force loss was lower over time after the pairedpulse rTMS intervention, indicating a possible effect of this intervention on muscle fatigue. These results indicated that the transient increase in cortical excitability after the rTMS protocol can, at least in part, compensate the loss of neural drive induced by central fatigue, i.e., "a progressive reduction of voluntary activation (VA) of muscle during exercise" (Gandevia, 2001).

This view has been further strengthened by several studies showing a positive effect of anodal transcranial direct current stimulation on different strength tasks (Cogiamanian et al., 2007; Tanaka et al., 2009; Williams et al., 2013). However, although the potential effects of non-invasive brain stimulation on central fatigue have been derived from behavioral studies, this has not yet been demonstrated directly with the twitch interpolated technique (Benwell et al., 2006; Cogiamanian et al., 2007). Moreover, the tasks performed have been single joint "laboratory tasks" and therefore the results cannot be directly transferred to "real world" multi-joint sport tasks.

The Wingate anaerobic test (WAnT) is a high intensity anaerobic sport specific test that seems to be ideally suited to examine performance and fatigue related modulations after rTMS (Coppin et al., 2012). Indeed, it has been shown that the WAnT is able to reduce drastically the force production of a following maximal isometric knee extension, and that this force reduction was associated with peripheral and central mechanisms (Fernandez-del-Olmo et al., 2013). Based on the impact of one WAnT on the another one, which was done 30 min later, the authors concluded that central rather than peripheral mechanisms might be responsible for a reduction in performance occurring after the WAnT (Fernandez-del-Olmo et al., 2013).

In the present study we hypothesized that an increased corticospinal excitability induced by rTMS will increase neural drive and performance in a sport specific task. Thus, we expected to find a higher power output during, and a higher resistance to central fatigue after the WAnT. For the rTMS treatment, we used the intermittent theta burst (iTBS) protocol, which was first described by Huang et al. (2005). To examine the potential effect of iTBS on central fatigue, we estimated the VA with TMS and peripheral nerve stimulation (PNS) in the quadriceps before and after the WAnT in two different sessions; one after real iTBS, the other after sham iTBS.

\section{MATERIALS AND METHODS}

\section{Subjects}

Ten regularly active males (mean/SD; age: 26 years, SD 2 years; weight: $78 \mathrm{~kg}$, SD $6 \mathrm{~kg}$; height: $181 \mathrm{~cm}, \mathrm{SD} 4 \mathrm{~cm}$ ) participated in the study, after giving written informed consent. The Ethics Committee at Konstanz Universität approved the study procedure. One part of the protocol was that subjects were deliberately kept naive about the existence of a placebo treatment. Contrariwise, they were informed that in the on going experiment two different treatment protocols were being tested. So, they were able to experience the difference between the protocols but throughout the whole study they were not aware of the existence of a control treatment.

\section{General Protocol}

Subjects participated in three familiarizations sessions and then in two experimental sessions consisting of a WAnT that was performed $5 \mathrm{~min}$ after either an iTBS or a control treatment. The experiment was conducted in counter balanced randomized order, i.e., five subjects started with the control treatment and five subjects with the iTBS treatment. $\mathrm{VA}_{\mathrm{TMS}}$, VA $\mathrm{A}_{\mathrm{PNS}}$, muscle twitch size at rest and motor evoked potentials (MEPs) of the right vastus lateralis $(\mathrm{VL})$ were measured three times prior to the iTBS treatment and four times after the WAnT (measurement starting 2 min after the end of the WAnT, see Figure 1A). Both sessions were separated by at least 2 (and at the most, 7) days of recovery. During neurophysiological measurements the subject was seated in a custom made chair with adjustable height and depth.

\section{Familiarization Sessions}

Prior to the two experimental sessions, the subjects participated in three familiarization sessions to get used to the WAnT, maximal voluntary contraction (MVC), TMS and PNS measurements. The three WAnTs reduced the bias induced by a possible training effect in the main study. At least 2 days and a maximum of 7 days separated each familiarization sessions.

\section{Wingate Anaerobic Test}

The WAnT was performed on a Cyclus2 ergometer (RBM elektronik-automation $\mathrm{GmbH}$, Leipzig, Germany). Just before each WAnT, a warm-up of $3 \mathrm{~min}$ at an intensity of $1 \mathrm{~W} / \mathrm{kg}$ body weight (BW) and a self-selected cadence was performed, interspersed with a seated sprint lasting 3-4 $\mathrm{s}$ that was started at the first and second minute of the warm-up. The WAnT was programmed for duration of $30 \mathrm{~s}$, starting at a cadence of $80 \mathrm{rpm}$, applying a constant force to the cranks automatically. Thus, the subjects were instructed to pedal at $60 \mathrm{rpm}$, and when they felt ready, to accelerate as fast as possible to the highest attainable frequency and to maintain it throughout the whole test duration while remaining in a seated position. The investigators ensured that the subject's motivation was maximal 


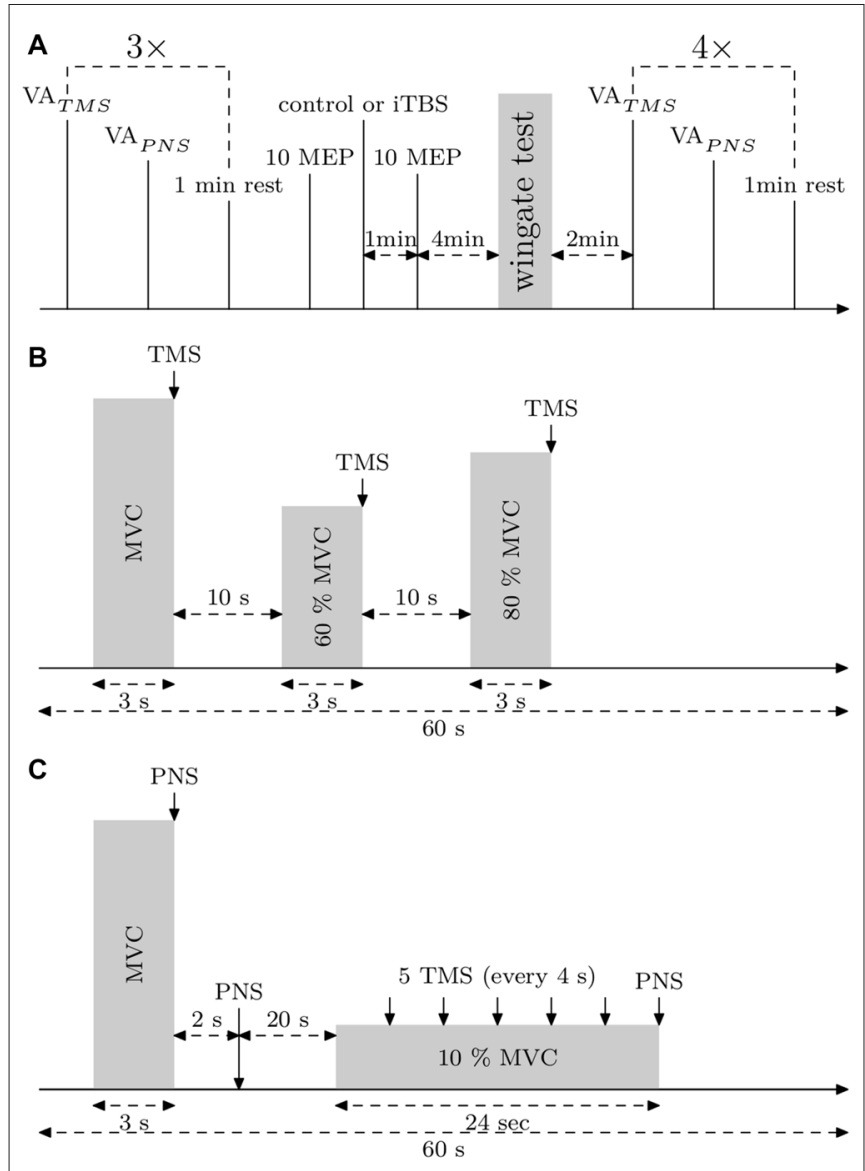

FIGURE 1 | Experimental protocols. (A) General protocol. Voluntary action (VA)TMS, VAPNS and motor evoked potentials (MEPS) (10\% maximal voluntary contraction [MVC]) were measured three times. Then, 10 MEPs (10\% MVC) were applied to control the basal corticospinal excitability. After that, the control or the intermittent theta burst (iTBS) treatment was carried out and 1 min later, 10 MEPs (10\% MVC) were applied. Then, the subject warmed up 3 min before the Wingate Anaerobic Test (WAnT). Immediately after the WAnT, the subject was fixed in the measurement chair and VATMS, VAPNS and MEPS at 10\% MVC were measured four times. (B) Protocol describing VA $A_{T M S}$ measurements. (C) Protocol describing VAPNS measurements and MEPs (10\% MVC) measurements.

and gave strong verbal encouragement throughout the $30 \mathrm{~s}$ test duration.

The resistive load (simulated flywheel mass) was individually adjusted using the following equation: Load $=(\text { Bodyweight })^{2} / 1000$. Mechanical data was sampled at a frequency of $8 \mathrm{~Hz}$. The power of the ergometer was calculated by the cyclus 2 software as followed: $P=2 * \mathrm{Pi}^{*} \mathrm{M} / \mathrm{Tr}$ where $\mathrm{M}$ is the torque at rear wheel in $\mathrm{Nm}$ and $\mathrm{Tr}$ the time for one rear wheel revolution in second. The overall error is SD $0.25 \%$ in the power range of $100-2000 \mathrm{~W}$. Peak power corresponds to the highest power output achieved during the $30 \mathrm{~s}$ test. Maximum cadence corresponds to the maximum pedalling rate reached during the $30 \mathrm{~s}$ test. Time-to-peak corresponds to the time needed to reach peak power, starting from the time point where a cadence of $80 \mathrm{rpm}$ was exceeded. Mean power is the average power calculated for the complete test duration. We calculated the fatigue index as the average decline in power per second from the time point where peak power was reached until the end of the test.

\section{EMG}

Bipolar surface electrodes (Bagnoli DE-2.1, DELSYS, Natick MA, USA; interelectrode distance: $10 \mathrm{~mm}$, electrode size: $1 \times 10 \mathrm{~mm}$ ) were applied over the muscle belly of the right VL, rectus femoris, vastus medialis and biceps femoris (BF) in the direction of the underlying muscle fibers. Beforehand, we shaved, sanded, and cleaned the skin with alcohol and placed the reference electrode over the right acromion. EMG signals were filtered (bandpass between 20 and $450 \mathrm{~Hz})$, amplified (*1k), sampled $(4000 \mathrm{~Hz})$ and registered on a computer with an analog-digital board (Micro 1401, CED, Cambridge Electronic Design Limited, Cambridge, England) for a posteriori analysis with Signal Software (CED Limited, Cambridge, England).

\section{Force Recordings}

The subjects were positioned in the chair with $90^{\circ}$ knee and hip angles. A non-compliant tension belt was fixed $\sim 2 \mathrm{~cm}$ above the right lateral malleolus (location was drawn on the skin for an exact repositioning pre and post WAnT) and connected to a force transducer (Model 9321A, Kistler, Winterthur, Switzerland) attached under the chair. Force signals from isometric quadriceps contractions were amplified and stored together with EMG data. A visual feedback of the current strength was displayed on a screen, with variation of scaling during the experiment and with the investigator indicating a target to reach $(10-20 \%$ over the MVC) as a supplementary motivational factor. During every contraction, the subject crossed his arms in front of his chest and was instructed to keep this position. The Investigator ensured that the subject's motivation was maximal during the whole study and gave a strong verbal encouragement throughout the MVC trials.

\section{Peripheral Nerve Stimulation (PNS)}

The cathode (custom made, $5 \mathrm{~cm}^{2}$, copper) was fixed on the femoral nerve in the femoral triangle and the anode (custom made, $24 \mathrm{~cm}^{2}$, bendable copper) on the center of the gluteus maximus. With this placement of the electrodes, PNS induced M-waves in the quadriceps without any muscular activity in the EMG of the BF. The locations of the electrodes were drawn on the skin for an exact replacement pre- and post-WAnT.

A Digitimer DS7A stimulator (Welwyn Garden City, UK) delivered single electrical stimuli (1 ms duration). The stimulus intensity to evoke Mmax was determined at rest (stimulation intensities required to reach Mmax were done between 20 and $60 \mathrm{~mA}$ depending on the subject). PNS intensity was set at $130 \%$ Mmax for supramaximal stimulations that were delivered in order to determine VA.

\section{Transcranial Magnetic Stimulation (TMS)}

Biphasic TMS pulses were delivered by a figure-of-eight coil, specifically designed to stimulate lower limb motor cortical area (MC-B70, MagVenture), and produced by a MagPro R30 
Stimulator (MagVenture). During the whole experiment, pulses were biphasic with the current flowing in the coil in an anterior-posterior/posterior-anterior direction. The coil was held so that the figure-of-eight was oriented perpendicularly to the interhemispheric fissure, with the center of the coil placed over the left hemisphere a few centimeters lateral of the vertex. The position was adjusted in order to elicit the biggest MEP possible in VL (around 40\% Mmax amplitude). The position of the coil was drawn on a swimming cap to ensure an identical position of the coil throughout the whole experiment. TMS intensity was set to elicit the biggest MEP possible during $\mathrm{VA}_{\mathrm{TMS}}$ measurements (90-100\% maximal stimulator output (MSO), identical intensity during the whole experiment) and a second intensity was used to produce MEPs equal to 10-20\% Mmax amplitude during $10 \%$ MVC. The intensity was then kept constant during the whole experiment.

\section{Control and iTBS Treatment}

Because the WAnT is an extremely demanding test, we have carefully chosen the iTBS treatment as a short and comfortable rTMS protocol (Cárdenas-Morales et al., 2010). The iTBS treatment we used was identical to the one described by Huang et al. (2005) with a stimulation intensity equal to $80 \%$ of active motor threshold obtained in the VL at 20\% MVC. The control treatment was identical to the iTBS treatment but with a maximal stimulation output of $2 \%$, which was enough to produce a clicking sound but definitely too low to induce any neural plasticity. During the control and iTBS treatment, the coil was placed over the intersection point between the interhemispheric fissure and the line passing through the hot spot and perpendicular to the interhemispheric fissure (see Figure 2B). The rationale behind this placement was to modify the excitability of the leg cortical area of both hemispheres, in order to change motor output in both legs. We used this protocol because the sequential application of iTBS can affect the excitability of the contralateral hemisphere (Suppa et al., 2008), which could invert the excitability of the second iTBS (MüllerDahlhaus and Ziemann, 2015). To control whether iTBS applied over the vertex have spreading effects over the leg cortical areas, we tested corticospinal excitability by stimulating the VL hotspot with TMS. Thus, 10 MEPs at 10\% MVC were measured a few minutes before and $1 \mathrm{~min}$ after the treatment. The average amplitude of the MEPs post-treatment was expressed in percentage of the average amplitude of the MEP pre-treatment. Immediately after the MEPs, the subject started to warm-up for the WAnT (see Figure 1A).

\section{Voluntary Activation}

VA, can be estimated by comparing the amplitude of a muscular twitch during a MVC caused by a supramaximal PNS or TMS over the motor cortex (superimposed twitch), with, the amplitude of the muscular twitch caused by the same stimulus in the potentiated muscle at rest (Gandevia et al., 1995) or the amplitude of the estimated twitch at rest (Todd et al., 2004). The measure of VA through PNS (VA $\mathrm{PNS}_{\mathrm{PN}}$ ) and VA through TMS (VA $\mathrm{VAMS}_{\mathrm{TM}}$ ) are used to demonstrate the existence of central and supraspinal fatigue respectively. Gandevia (2001) defined central fatigue as a "reduction in VA of muscle during exercise" and supraspinal fatigue as "fatigue produced by failure to generate output from the motor cortex". Here, supraspinal fatigue is considered as a subset of central fatigue and although they cannot be directly compared mainly due to methodological restrictions (e.g., activation of different muscles during TMS compared to PNS), they can give an insight regarding the possible location of fatigue (Todd et al., 2003).

Before VA measurements, the subject warmed up 10 min with incremental isometric contractions. During VA measurements, when there was no visible plateau in MVC, or when the timing of the stimulation was not correct, or if the subject revealed it was not an MVC, the trial was rejected and repeated after 20-30 s. This case was seen only for two subjects during the pre measurements, for whom, maybe, the preceding warmup was not enough, hence one or two more trials to reach real MVC and real VA. Stimulations were triggered manually during the plateau of the MVC. To assess VA $\mathrm{T}_{\mathrm{TMS}}$ we used a protocol according to Todd et al. (2004) and Sidhu et al. (2009). The estimated twitch at rest is estimated from a linear regression between the size of the superimposed twitches obtained during MVC, 60 and 80\% MVC contractions (see Figure $1 \mathrm{~B}$ ) and the level of voluntary contraction. $\mathrm{VA}_{\mathrm{TMS}}$ was defined as: $\mathrm{VA}_{\mathrm{TMS}}=(1-$ superimposed twitch/estimated twitch at rest) $\times 100$.

VAPNS was defined as: VAPNS $(1-$ superimposed twitch/resting twitch) $\times 100$, where the superimposed twitch is elicited with a supramaximal PNS during MVC and the resting twitch by PNS, $2 \mathrm{~s}$ after the MVC (see Figure 1C). VAPNS was measured $1 \mathrm{~min}$ after $\mathrm{VA}_{\mathrm{TMS}}$ and there was $1 \mathrm{~min}$ of rest before the next cycle (see Figure 1A). The best out of the 3 VA measurements performed before the WAnT was taken as the pre WAnT VA value (as depicted in Figure 3).

\section{Corticospinal Excitability}

To assess corticospinal excitability, 5 MEPs at 10\% MVC (with interstimulus intervals set at $4 \mathrm{~s}$ ) were measured starting $20 \mathrm{~s}$ after VAPNs (see Figure 1C). The amplitude of each MEP was normalized to the amplitude of the following Mmax elicited at $10 \%$ MVC.

\section{Maximal Voluntary Contraction Before and After the Task}

We measured the maximal force (MVC) during the VA $\mathrm{A}_{\mathrm{TMS}}$ measurements pre- and post-WAnT, with and without iTBS, and expressed the MVC in Newtons.

\section{Time Course Effect of iTBS on Corticospinal Excitability}

We performed an additional experiment on six subjects to verify that the iTBS protocol we used in the present study was able to increase the corticospinal excitability substantially (see Figure 2A). In this additional experiment we modified the time delay from the end of the iTBS treatment and the TMS 


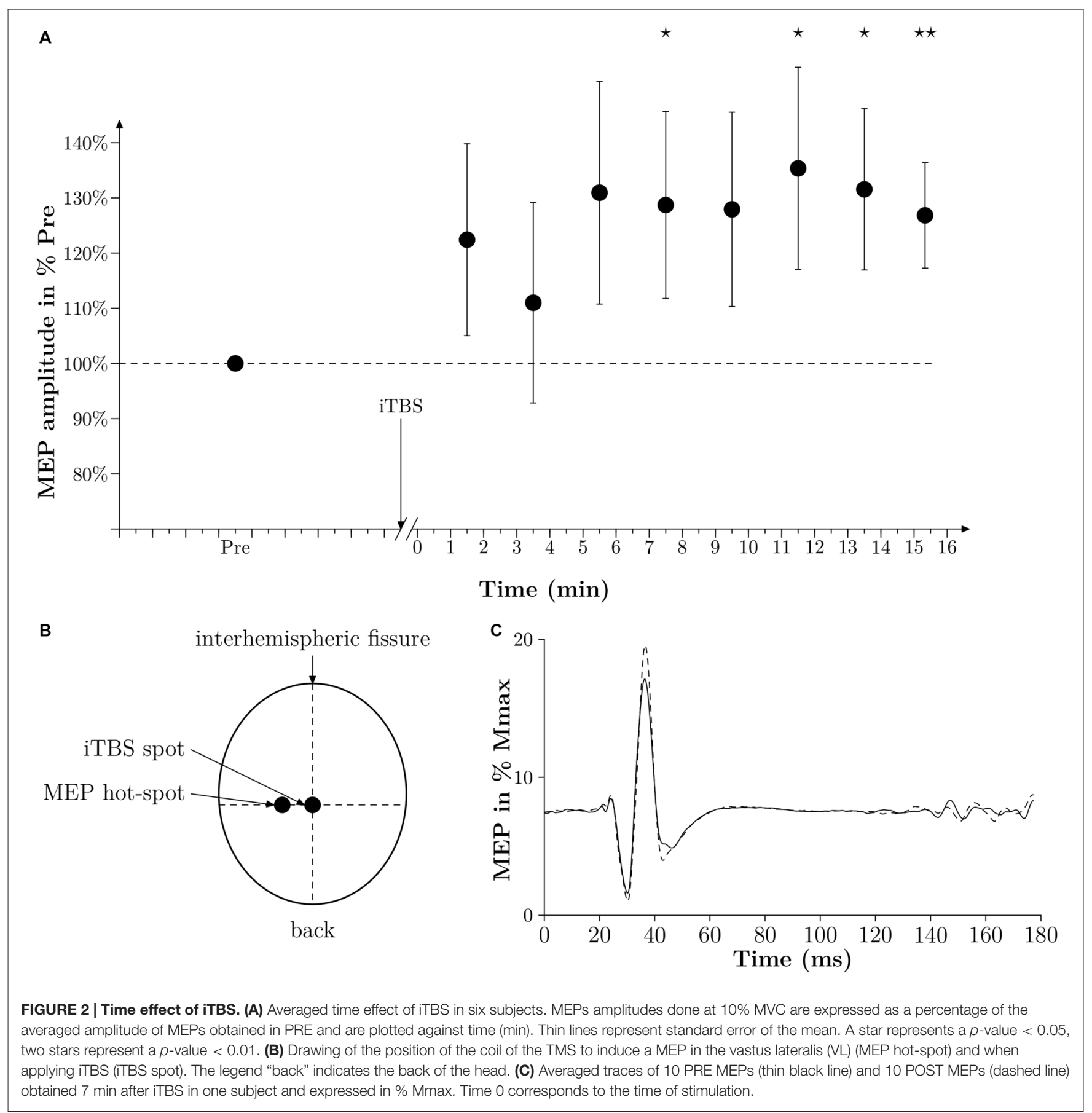

measurements. With this protocol we were then able to follow the changes in MEPs from 1 min after iTBS until 15 min after iTBS. At the beginning of the experiment, the subject had to warm up with isometric contractions. MVC was assessed after three maximum trials separated by 1 min of rest. The intensity of TMS was set so the MEP amplitude during a contraction of $10 \%$ MVC was equal to $10-20 \%$ of the Mmax amplitude, which had already been determined at 10\% MVC beforehand. One set of MEPs consisted of 10 MEPs induced every $4 \mathrm{~s}$. One set was done $5 \mathrm{~min}$ before iTBS (PRE), and then $1 \mathrm{~min}$ after sets were done every $1 \mathrm{~min} 20 \mathrm{~s}$ for $15 \mathrm{~min}$. MEP amplitudes were expressed in percentages of the averaged MEP amplitude obtained in PRE.

\section{Statistical Analysis}

All statistical tests were done with R (3.1.0, copyright 2014, The R Foundation for Statistical Computing Platform). Twotailed paired $t$-tests were conducted to compare peak power, time-to-peak, mean power, maximum cadence, fatigue index, and pre- and post-treatment MEP amplitude. Unilateral one sample $t$-tests were conducted on MEP amplitudes post-iTBS 
(time course effect of iTBS experiment) to show a possible difference with a value of 100 (i.e., PRE value). Two ways within subject ANOVAs were applied for muscle twitch size at rest, VA $A_{P N S}, \mathrm{VA}_{\mathrm{TMS}}, \mathrm{MVC}, \mathrm{MEP}$ at $10 \% \mathrm{MVC}, \mathrm{Mmax}$ during MVC, Mmax during 10\% MVC and Mmax at rest to detect time effects, treatment effects, and time $\times$ treatment interaction effects. Post hoc tests were done with pairwise comparisons and a Bonferroni correction. When an ANOVA showed a time effect, we made comparisons only for the factor time. When an interaction was shown, we made comparisons for one factor with the other factor fixed and vice versa, i.e., comparisons on the different time point for each treatment and comparison between treatments for each time point.

Retrospective power analysis was applied for the first measurement of $\mathrm{VA}_{\mathrm{TMS}}, \mathrm{VA}_{\mathrm{PNS}}$, potentiated twitches, MEP and MVC done after the WAnT with sham or iTBS treatment. Power calculations were done for a paired $t$-test, with an effect size represented by the Cohen's $d$ and a statistical significance of 0.05 .

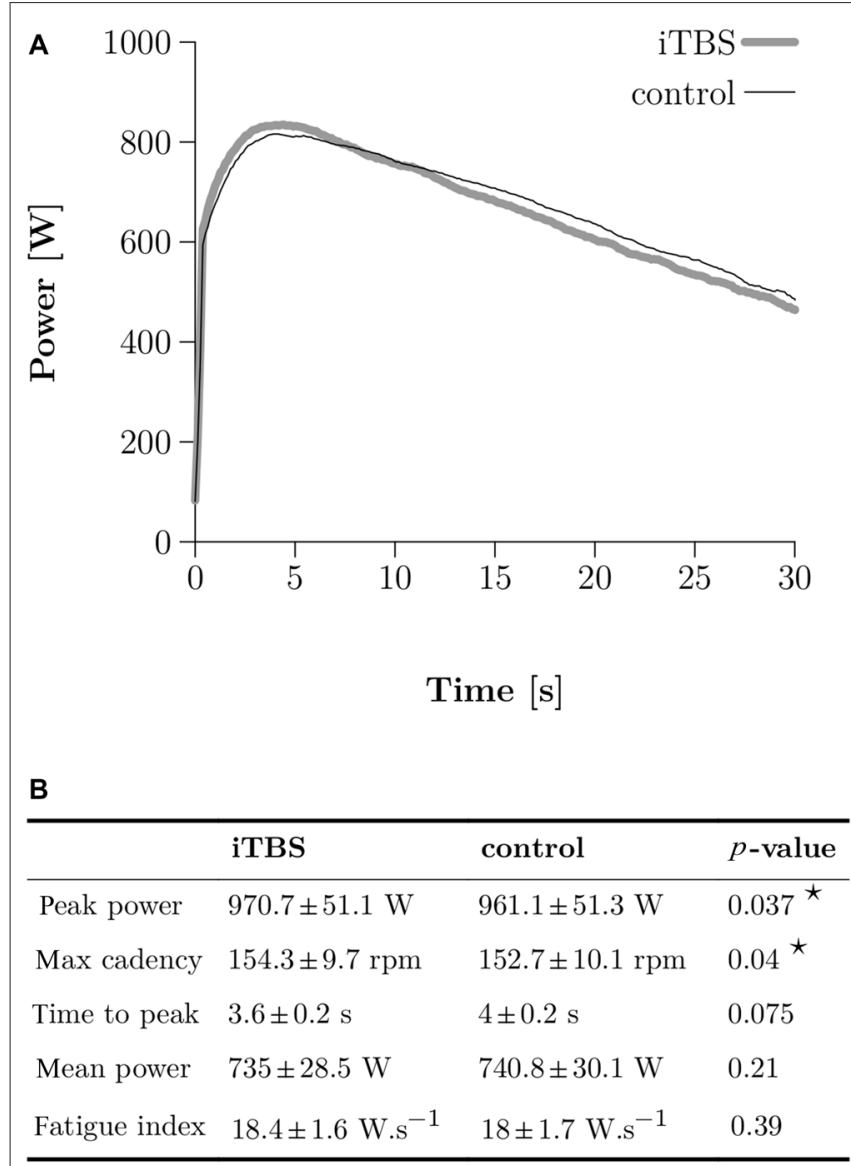

FIGURE 3 | Wingate Anaerobic Test (WAnT) results. (A) Two typical WAnT power curves obtained in one single subject after control (thin black line) and iTBS treatment (thick gray line). (B) Group data $(N=10)$ for the most important parameters, which characterize the power development in the WAnT. Bilateral paired $t$-tests were applied and a star corresponds to a significant result $(p<0.05)$

\section{RESULTS}

The typical power curve obtained during a WAnT after either iTBS or control in one subject is depicted in Figure 3A. A twotailed paired $t$-test demonstrated higher peak power in the WAnT after iTBS compared to the controls (961 SE $51 \mathrm{~W}$ for control and 970 SE $7 \mathrm{~W}$ for iTBS, $p=0.037$; all numbers following SE correspond to the standard error of the mean) as well as a higher max cadence (153 SE $3 \mathrm{rpm}$ for control and 154 SE $3 \mathrm{rpm}$ for iTBS, $p=0.04)$. We found no significant differences in mean power, fatigue index, and time to reach the peak power (see Figure 3B).

Moreover, we could not observe a significant difference in the amplitude of the 10 MEPs measured before, and $1 \mathrm{~min}$ after, the treatment irrespective of the treatment itself (108 SE $6 \%$ for control and 112 SE 5\% for iTBS, $p=0.64$ ). However, as depicted in Figures $\mathbf{2 A}, \mathbf{C}$, iTBS significantly increased the MEP amplitude by $28 \% 7 \mathrm{~min}$ after the treatment. It must be noted that in this complementary experiment the time-window with increased MEPs corresponds to the period of WAnT testing in the main experiments. It is also remarkable that during this complementary experiment, however, the iTBS treatment had no visible effect in two out of the six subjects tested.

As presented in Figure 4, the ANOVAs showed time effects, but no treatment and time $\times$ treatment effects, for the potentiated twitches elicited with PNS at rest $(p=0.001$, $p=0.261$ and $p=0.897$, respectively), as well as for VAPNS ( $p=0.015, p=0.462$ and $p=0.906$, respectively). No significant effects were seen for the MEP amplitudes $(p=0.938$, $p=0.591$ and $p=0.432$ ). For MVC measured during $\mathrm{VA}_{\mathrm{TMS}}$ we observed a time and time $\times$ treatment effect $(p \leq 0.001$ and $p=0.025$, respectively) but no treatment effect $(p=0.72$ ). Post hoc tests showed: (i) a difference between PRE and Post 2 ( $p=0.019)$, PRE and Post $3(p=0.02)$ for the control treatment. (ii) A difference between PRE and Post $1(p \leq 0.001)$, PRE and Post $2(p=0.029)$, Post 1 and Post $3(p=0.025)$ and Post 1 and Post $4(p \leq 0.001)$ for the iTBS treatment. For $V_{\text {TMS }}$ we observed a time effect as well as a time $\times$ treatment effect ( $p=0.002$ and $p=0.001$, respectively), but no effect for treatment alone $(p=0.286)$. Post hoc tests showed differences between PRE and Post $1(p \leq 0.001)$, PRE and Post $4(p \leq 0.001)$ and between Post 1 and Post $3(p=0.008)$ for the control treatment. Moreover, there was a difference between treatments at PRE $1(p \leq 0.001)$.

As displayed in Figure 5, there were no effects of time and treatment on Mmax measured during $\operatorname{MVC}(p=0.06,0.10$ and 0.41 for time, treatment and time $\times$ treatment respectively), during $10 \% \operatorname{MVC}(p=0.98,0.84$ and 0.50$)$ and at rest $(p=0.11$, 0.49 and 0.96 ).

Retrospective power analysis applied on the first measurement done after the WAnT and comparing the values obtained with sham or iTBS treatment were done for $\mathrm{VA}_{\mathrm{TMS}}$ (Cohen's $d=1.29$, power $=0.95$ ), VAPNS (Cohen's $d=0.32$, power $=0.15$ ), potentiated twitch at rest (Cohen's $d=0.15$, power $=0.07)$, MEP (Cohen's $d=0.25$, power $=0.11)$ and MVC (Cohen's $d=0.35$, power $=0.17$ ). 


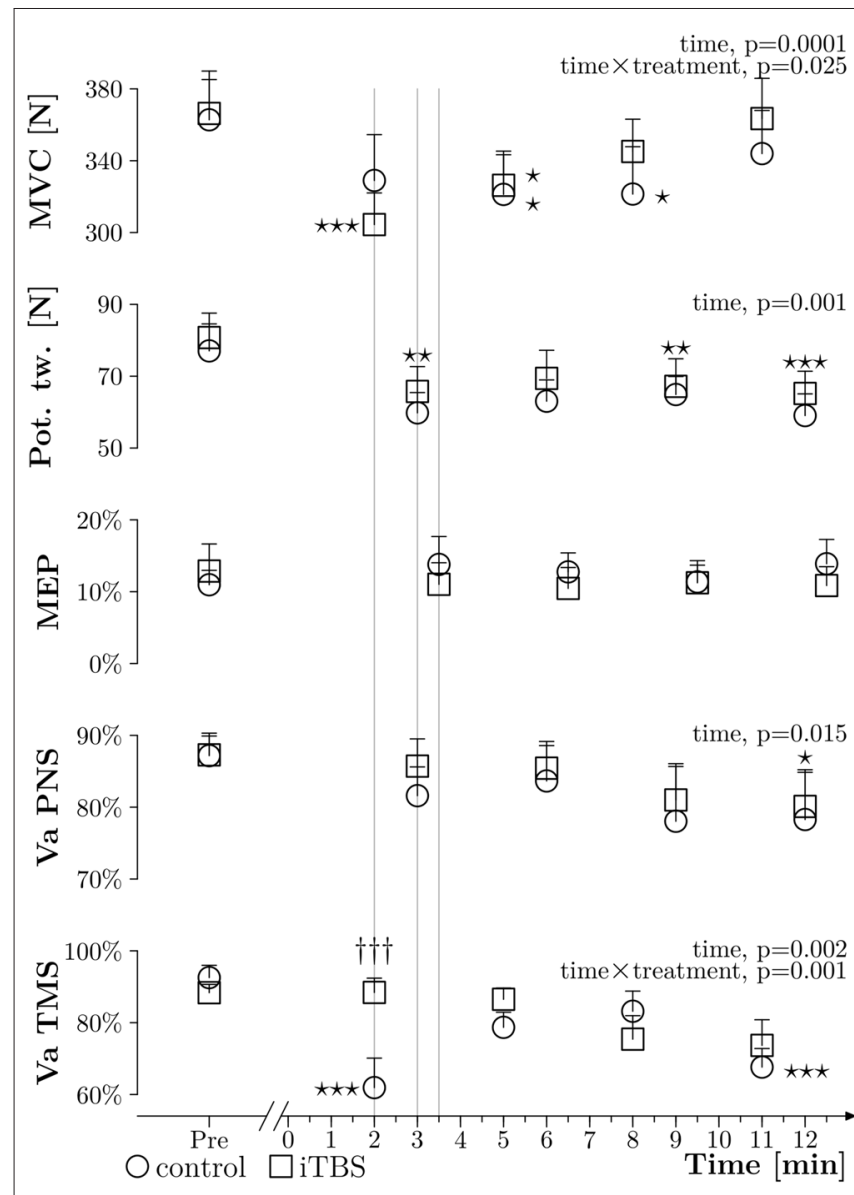

FIGURE 4 | General results. Grouped data $(N=10)$ about MVC obtained during VATMS (in N), amplitude of potentiated twitches at rest (expressed in N), MEPs measured during 10\% MVC (expressed in \% Mmax), VAPNS and VA TMS plotted against time, before (PRE) and after the WAnT ( 0 min corresponds to the end of the WAnT), with the control treatment (circles) and with the iTBS treatment (squares); thin lines represent the standard error of the mean. The three thin gray vertical lines highlight differences in timing between measurements. Two way ANOVAs within subjects were applied and significant results are described on the right side of each curve. Stars correspond to a significant difference (one star: $p<0.05$, two stars: $p<0.01$ and three stars: $p<0.001)$ related to time effect. Daggers represent a treatment difference for a time point $(p<0.001)$.

\section{DISCUSSION}

The present study has shown for the first time that iTBS, applied 5 min prior to a WAnT, could improve peak power and maximum pedalling cadence, and could suppress the subsequent supraspinal fatigue normally observed in the quadriceps muscles in healthy subjects during a high intensity sport specific task. However, the suppression of supraspinal fatigue was not accompanied by a higher performance during the later phases of the task.

\section{The Effect of iTBS on WAnT}

The increase of peak power observed after the iTBS treatment is statistically significant but very small ( $1 \%$ increase). It has to be acknowledged that this difference between the treatments

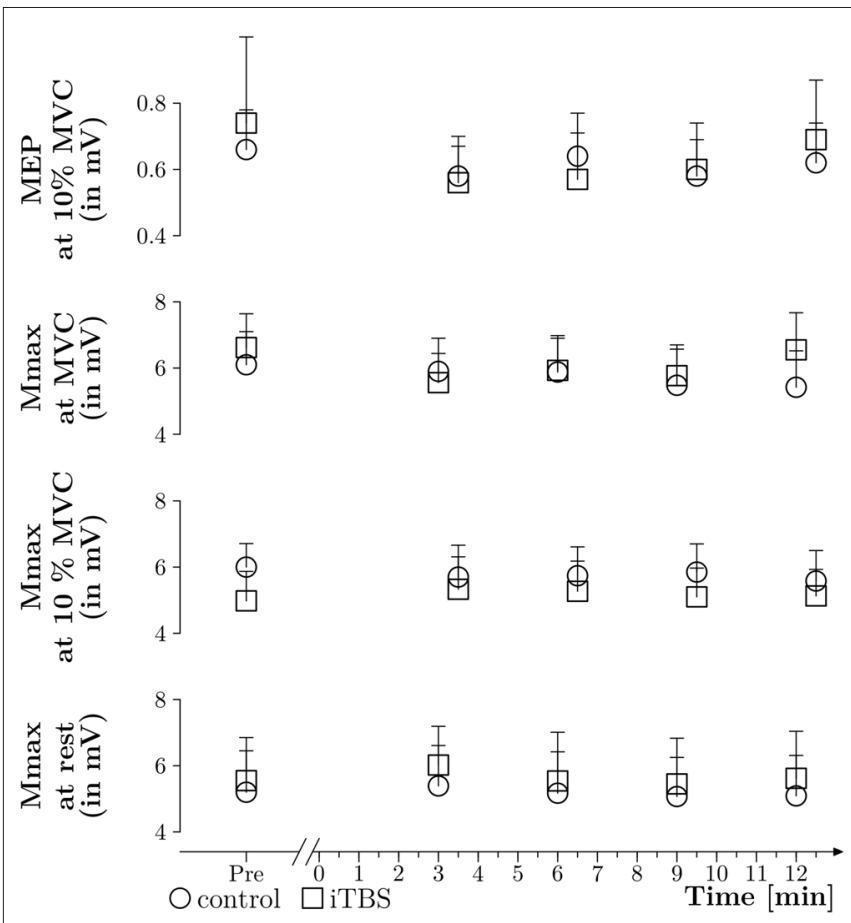

FIGURE 5 | Mmax and MEP absolute values. Absolute amplitudes of MEPs were measured during 10\% MVC contraction. The TMS intensity was set in order to obtain MEP equal to $10-20 \%$ of Mmax before the WAnT and then kept constant throughout the experiment. Mmax was elicited during 10\% MVC contraction, at rest in the potentiated muscle (obtained during VApNS procedure) and during MVC (also obtained during VAPNS procedure). All values are expressed in $\mathrm{mV}$ and the thin lines correspond to the standard error of the mean.

could come from a placebo effect or even a statistical alpha error, and seems to be too small to be of applied relevance. However, when taking into account the context of the task, this result seems plausible and relevant. Firstly, an increase in power was seen in 7 out of the 10 subjects ( 2 subjects experienced a decrease in power and one had no modifications). Secondly, when considering the improvements in the peak power seen between the three familiarization sessions, an increase of $1 \%$ is performance relevant: an increase of $2.1 \%$ between session 1 and session 2 (from $919.8 \mathrm{~W}$ to $944.8 \mathrm{~W}$ ), and an increase of $0.9 \%$ between session 2 and session 3 (from 944.8 W to $953.4 \mathrm{~W}$ ). Thus, the iTBS protocol used in the present study seems to be potentially relevant in increasing performance in a maximal anaerobic cycling exercise. We believe that further research in this direction and especially toward the optimization of training throughout a whole sport season could bring new interesting insights in the actual training concepts.

Interestingly, the differences in performance seem to be restricted to peak power and maximal cadence, as we were not able to show any differences in mean power or in the fatigue index of a WAnT. This rather specific effect may be explained by an increased neural drive induced by iTBS. An enhanced neural drive, mainly because of an increased excitability of the excitatory synaptic inputs to pyramidal neurones after iTBS (Di Lazzaro et al., 2008) might modify the efficiency of motor neuronal 
recruitment (Kernell and Hultborn, 1990), which would allow an easier recruitment of large motoneurons. This enhanced neural drive may also increase the frequency of discharge of motoneurons (Desmedt and Godaux, 1977), and consequently may increase the force production of the discharging motor units (Mrówczyński et al., 2015). Both mechanisms would increase muscular power and the pedalling cadence at the beginning of the maximal cycling task, but do not necessarily affect power over the entire period of the task or the following MVCs.

An alternative explanation for the enhancement of peak power could come from an increased motor learning effect due to the iTBS protocol (Teo et al., 2011). Indeed, during the warm up, which was done right after the iTBS treatment, the subjects had to increase their cadence on two occasions, as quickly as possible. However, in our case, this hypothesis seems unlikely due to the three previous familiarization sessions, which were made to specifically reduce a potential bias arising from motor learning.

Moreover, it seems unlikely that the suppression of supraspinal fatigue with the iTBS treatment directly influenced the peak power and the maximal cadence, as it has been reported that during a maximal $5 \mathrm{~s}$ cycling sprint, the EMG activity of the muscles mainly responsible for overall power in the task (such as the VL) doesn't change, which suggests an as yet unaltered VA at the time you reach peak power (Hautier et al., 2000).

\section{Suppression of Supraspinal Fatigue Without Changes in Fatigue Related Performance}

In the present study supraspinal fatigue after iTBS was considerably lower compared to control. It must be noted that our control measurements are in line with previous results. Indeed, in our study, $\mathrm{VA}_{\mathrm{TMS}}$ went from $92 \%$ to $61 \%$ after the WAnT, and in the study of Fernandez-del-Olmo et al. (2013), $\mathrm{VA}_{\mathrm{TMS}}$ went from $89 \%$ to $62 \%$. This high reproducibility of control VA $\mathrm{VA}_{\mathrm{TMS}}$ measurements is a strong indicator that iTBS removed a part of the supraspinal fatigue $(88 \%$ after the iTBS protocol vs. $61 \%$ after the control protocol).

Given this significant difference in supraspinal fatigue the question arises: why couldn't we observe any changes in fatigue related performance? A less-impaired VA should, in theory, allow higher muscle force and power throughout the fatiguing task. Thus, we would expect a higher mean power during the WAnT, as well as a higher MVC after the WAnT, which we were not able to show in the present study. Three possible explanations arise.

Firstly, as we did not measure $\mathrm{VA}_{\mathrm{TMS}}$ during the WAnT, it is possible that iTBS did not affect the reduction of $\mathrm{VA}_{\mathrm{TMS}}$ during the task, but only affected its recovery after the end of the task. This would explain the lack of any fatigue related performance differences between the iTBS and control treatments and the increased VA $\mathrm{A}_{\mathrm{TMS}} 2 \mathrm{~min}$ after the end of the WAnT. However, this hypothesis does not explain the presence of a low MVC despite a high VA $\mathrm{T}_{\mathrm{TMS}}$.

Secondly, it could be that the systemic stress and the peripheral fatigue induced by the WAnT masked any potential beneficial effects of a less-impaired $\mathrm{VA}_{\mathrm{TMS}}$ on fatigue related performance. Indeed, the overall performance during MVC is dependent of both, VA and the ability of the muscle to produce force. The weakest link of the chain then may limit overall performance to the greatest extent. Hence, the result of significantly lower MVC after the WAnT despite high VA after the iTBS treatment in comparison with control may be explained by peripheral fatigue limiting the force output during MVC and presumably also during the WAnT; perhaps as a sub-product of the greater performance in the WAnT induced by real iTBS. This assumption could also explain the difference in the results of our study and the study of Benwell et al. (2006) where the authors didn't report an increase in force after rTMS, but rather reported a decreased rate of force loss. Indeed, the WAnT is a whole body power exercise and induces a very large amount of systemic stress (Baker et al., 2002; Coppin et al., 2012), whereas a pinch grip is more of a strength task that requires the use of only a few small muscles with a negligible systemic impact. This implies that the limitation of the performance and the failure of the task may not be induced by the same components of fatigue, or at least not in the same proportions, and that the excitability of motor structures could be affected differently (for review, see Gandevia, 2001; Gruet et al., 2013).

Thirdly, central networks that contribute to the overall motor drive but are not located within the corticospinal tract and cannot be assessed with VA $\mathrm{TMS}_{\mathrm{TM}}$, may also impair MVC.

\section{Discrepancies Between VATMS and VAPNS Measurements}

Although it is difficult to make direct comparisons between $\mathrm{VA}_{\text {TMS }}$ and VAPNs (Todd et al., 2003), supraspinal fatigue is a subset of central fatigue, and thus, after exercise, the decrease of $\mathrm{VA}_{\text {TMS }}$ should, in theory, not be independent of the decrease of VAPNS (Taylor et al., 2006). However, in our study, after the WAnT, VA $\mathrm{V}_{\text {TMS }}$ was impaired but not VAPNS, and the iTBS treatment significantly influenced $\mathrm{VA}_{\mathrm{TMS}}$ but not $\mathrm{VA}_{P N S}$. In our opinion, the discrepancies between the $\mathrm{VA}_{\mathrm{TMS}}$ and VAPNS are mainly related to the timing of the measurements in the present study. Indeed, it is well known that recovery from central fatigue in the quadriceps can be very fast (Gruet et al., 2014). Assuming a steep recovery course for central fatigue after the WAnT, it seems possible that we were able to measure a significant lower $\mathrm{VA}_{\text {TMS }} 2$ min after the end of the WAnT but failed to measure a reduced VAPNS $3 \mathrm{~min}$ after (see Figure 3 ). It must be noted that we decided to separate VA $\mathrm{A}_{\mathrm{TMS}}$ and VAPNS measurements by $1 \mathrm{~min}$ in order to limit the influence of the first measurement on the second one. Indeed, we were not sure that many subjects could truly reach two times MVC without a break of $1 \mathrm{~min}$, due to the acute stress induced by the WAnT.

\section{Limitations of the Study}

Based on the relevant literature, it is rather unusual to deliver an iTBS treatment with the stimulation coil placed over the cortical leg motor area of both hemispheres. We were able to show in an additional experiment (see Figure 2A) that effects on MEPs were similar compared to results in previous 
studies that placed the coil over the hot-spot of one hemisphere (Huang et al., 2005). Nevertheless, its effects on the neuronal networks regulating the motor output of both sides, and on the interaction of both hemispheres are not known. Another problematic point regarding the iTBS treatment lies in the fact that the control treatment (TMS at 2\% MSO) and the real iTBS treatment could obviously be discerned by the subjects. To compensate this issue, the subjects were unaware of the existence of a real treatment and a placebo treatment, and thus not aware of the fact that one treatment only may have a physiological effect. Nevertheless, it cannot be excluded that the difference in peak power between the two groups may be influenced more by a placebo effect rather than by a physiological effect.

The other main limitation comes from the delay of measurements of VAPNS as addressed in the section above. Indeed, with such a long latency, it is probable that a considerable part of central fatigue which exists right after the end of the task can't be observed anymore due to a fast recovery already $2 \mathrm{~min}$ later (Gruet et al., 2014). To reduce the time delay between the end of the fatiguing task and the $\mathrm{VA}_{\mathrm{TMS}}$ and $\mathrm{VA}_{\mathrm{PNS}}$ measurements we suggest to include only participants with a very good physical condition who are able to compensate the discomfort and stress and immediately perform

\section{REFERENCES}

Baker, J., Brown, E., Hill, G., Phillips, G., Williams, R., and Davies, B. (2002). Handgrip contribution to lactate production and leg power during high-intensity exercise. Med. Sci. Sports Exerc. 34, 1037-1040. doi: 10. 1097/00005768-200206000-00021

Benwell, N. M., Mastaglia, F. L., and Thickbroom, G. W. (2006). Pairedpulse rTMS at trans-synaptic intervals increases corticomotor excitability and reduces the rate of force loss during a fatiguing exercise of the hand. Exp. Brain Res. 175, 626-632. doi: 10.1007/s00221-006-0579-3

Cárdenas-Morales, L., Nowak, D. A., Kammer, T., Wolf, R. C., and SchönfeldtLecuona, C. (2010). Mechanisms and applications of theta-burst rTMS on the human motor cortex. Brain Topogr. 22, 294-306. doi: 10.1007/s10548-0090084-7

Carey, J. R., Fregni, F., and Pascual-Leone, A. (2006). rTMS combined with motor learning training in healthy subjects. Restor. Neurol. Neurosci. 24, 191-199.

Censor, N., and Cohen, L. G. (2011). Using repetitive transcranial magnetic stimulation to study the underlying neural mechanisms of human motor learning and memory. J. Physiol. 589, 21-28. doi: 10.1113/jphysiol.2010. 198077

Cogiamanian, F., Marceglia, S., Ardolino, G., Barbieri, S., and Priori, A. (2007). Improved isometric force endurance after transcranial direct current stimulation over the human motor cortical areas. Eur. J. Neurosci. 26, 242-249. doi: 10.1111/j.1460-9568.2007.05633.x

Coppin, E., Heath, E. M., Bressel, E., and Wagner, D. R. (2012). Wingate anaerobic test reference values for male power athletes. Int. J. Sports Physiol. Perform. 7, $232-236$.

Desmedt, J. E., and Godaux, E. (1977). Ballistic contractions in man: characteristic recruitment pattern of single motor units of the tibialis anterior muscle. J. Physiol. 264, 673-693. doi: 10.1113/jphysiol.1977.sp011689

Di Lazzaro, V., Pilato, F., Dileone, M., Profice, P., Oliviero, A., Mazzone, P., et al. (2008). The physiological basis of the effects of intermittent theta burst stimulation of the human motor cortex. J. Physiol. 586, 3871-3879. doi: 10. 1113/jphysiol.2008.152736

Fernandez-del-Olmo, M., Rodriguez, F. A., Marquez, G., Iglesias, X., Marina, M., Benitez, A., et al. (2013). Isometric knee extensor fatigue following a Wingate at there maximum very shortly after the end of the fatiguing exercise.

\section{CONCLUSION}

We have shown increased peak power and maximum pedalling cadence during a WAnT, and increased VA measured with TMS, which could be interpreted as a suppression of supraspinal fatigue after an iTBS treatment. However, variables related to the performance over the entire workout period were not affected despite a significant suppression of supraspinal fatigue. This work can be seen as a first indication that non-invasive brain stimulation methods could have a relevancy regarding the optimization of the performance in sport specific tasks.

\section{FUNDING}

This study was conducted without any external funding.

\section{AUTHOR CONTRIBUTIONS}

L-SG and MG designed the study. L-SG, PT and RB collected and analyzed the data. L-SG interpreted the data. L-SG and MG drafted the manuscript.

test: peripheral and central mechanisms. Scand. J. Med. Sci. Sports 23, 57-65. doi: 10.1111/j.1600-0838.2011.01355.x

Gandevia, S. C. (2001). Spinal and supraspinal factors in human muscle fatigue. Physiol. Rev. 81, 1725-1789.

Gandevia, S. C., Allen, G. M., and Mckenzie, D. K. (1995). Central fatigue. Critical issues, quantification and practical implications. Adv. Exp. Med. Biol. 384, 281-294. doi: 10.1007/978-1-4899-1016-5_22

Gruet, M., Temesi, J., Rupp, T., Levy, P., Millet, G. Y., and Verges, S. (2013). Stimulation of the motor cortex and corticospinal tract to assess human muscle fatigue. Neuroscience 231, 384-399. doi: 10.1016/j.neuroscience.2012.10.058

Gruet, M., Temesi, J., Rupp, T., Levy, P., Verges, S., and Millet, G. Y. (2014). Dynamics of corticospinal changes during and after high-intensity quadriceps exercise. Exp. Physiol. 99, 1053-1064. doi: 10.1113/expphysiol.2014.078840

Hautier, C. A., Arsac, L. M., Deghdegh, K., Souquet, J., Belli, A., and Lacour, J. R. (2000). Influence of fatigue on EMG/force ratio and cocontraction in cycling. Med. Sci. Sports Exerc. 32, 839-843. doi: 10.1097/00005768-200004000-00017

Hoogendam, J. M., Ramakers, G. M., and Di Lazzaro, V. (2010). Physiology of repetitive transcranial magnetic stimulation of the human brain. Brain Stimul. 3, 95-118. doi: 10.1016/j.brs.2009.10.005

Hortobagyi, T., Richardson, S. P., Lomarev, M., Shamim, E., Meunier, S., Russman, H., et al. (2009). Chronic low-frequency rTMS of primary motor cortex diminishes exercise training-induced gains in maximal voluntary force in humans. J. Appl. Physiol. (1985) 106, 403-411. doi: 10.1152/japplphysiol.90701. 2008

Huang, Y. Z., Edwards, M. J., Rounis, E., Bhatia, K. P., and Rothwell, J. C. (2005) Theta burst stimulation of the human motor cortex. Neuron 45, 201-206. doi: 10.1016/j.neuron.2004.12.033s

Kakuda, W., Abo, M., Watanabe, S., Momosaki, R., Hashimoto, G., Nakayama, Y., et al. (2013). High-frequency rTMS applied over bilateral leg motor areas combined with mobility training for gait disturbance after stroke: a preliminary study. Brain Inj. 27, 1080-1086. doi: 10.3109/02699052.2013.794973

Kernell, D., and Hultborn, H. (1990). Synaptic effects on recruitment gain: a mechanism of importance for the input-output relations of motoneurone pools? Brain Res. 507, 176-179. doi: 10.1016/0006-8993(90)90542-j

Khedr, E. M., Rothwell, J. C., Shawky, O. A., Ahmed, M. A., and Hamdy, A. (2006). Effect of daily repetitive transcranial magnetic stimulation on motor 
performance in Parkinson's disease. Mov. Disord. 21, 2201-2205. doi: 10. 1002/mds.21089

Lefaucheur, J. P., André-Obadia, N., Antal, A., Ayache, S. S., Baeken, C., Benninger, D. H., et al. (2014). Evidence-based guidelines on the therapeutic use of repetitive transcranial magnetic stimulation (rTMS). Clin. Neurophysiol. 125, 2150-2206. doi: 10.1016/j.clinph.2014.05.021

Lefaucheur, J. P., Drouot, X., Von Raison, F., Ménard-Lefaucheur, I., Cesaro, P., and Nguyen, J. P. (2004). Improvement of motor performance and modulation of cortical excitability by repetitive transcranial magnetic stimulation of the motor cortex in Parkinson's disease. Clin. Neurophysiol. 115, 2530-2541. doi: 10.1016/j.clinph.2004.05.025

Mrówczyński, W., Celichowski, J., Raikova, R., and Krutki, P. (2015). Physiological consequences of doublet discharges on motoneuronal firing and motor unit force. Front. Cell. Neurosci. 9:81. doi: 10.3389/fncel.2015. 00081

Muellbacher, W., Ziemann, U., Boroojerdi, B., and Hallett, M. (2000). Effects of low-frequency transcranial magnetic stimulation on motor excitability and basic motor behavior. Clin. Neurophysiol. 111, 1002-1007. doi: 10.1016/s13882457(00)00284-4

Müller-Dahlhaus, F., and Ziemann, U. (2015). Metaplasticity in human cortex. Neuroscientist 21, 185-202. doi: 10.1177/1073858414526645

Palm, U., Ayache, S. S., Padberg, F., and Lefaucheur, J. P. (2014). Non-invasive brain stimulation therapy in multiple sclerosis: a review of tDCS, rTMS and ECT results. Brain Stimul. 7, 849-854. doi: 10.1016/j.brs.2014.09.014

Ridding, M. C., and Rothwell, J. C. (2007). Is there a future for therapeutic use of transcranial magnetic stimulation? Nat. Rev. Neurosci. 8, 559-567. doi: 10. 1038/nrn2169

Schlaghecken, F., Münchau, A., Bloem, B. R., Rothwell, J., and Eimer, M. (2003). Slow frequency repetitive transcranial magnetic stimulation affects reaction times, but not priming effects, in a masked prime task. Clin. Neurophysiol. 114, 1272-1277. doi: 10.1016/s1388-2457(03)00118-4

Sidhu, S. K., Bentley, D. J., and Carroll, T. J. (2009). Cortical voluntary activation of the human knee extensors can be reliably estimated using transcranial magnetic stimulation. Muscle Nerve 39, 186-196. doi: 10.1002/mus.21064

Suppa, A., Ortu, E., Zafar, N., Deriu, F., Paulus, W., Berardelli, A., et al. (2008). Theta burst stimulation induces after-effects on contralateral primary motor cortex excitability in humans. J. Physiol. 586, 4489-4500. doi: 10.1113/jphysiol. 2008.156596

Tanaka, S., Hanakawa, T., Honda, M., and Watanabe, K. (2009). Enhancement of pinch force in the lower leg by anodal transcranial direct current stimulation. Exp. Brain Res. 196, 459-465. doi: 10.1007/s00221-009-1863-9

Taylor, J. L., Todd, G., and Gandevia, S. C. (2006). Evidence for a supraspinal contribution to human muscle fatigue. Clin. Exp. Pharmacol. Physiol. 33, 400-405. doi: 10.1111/j.1440-1681.2006.04363.x
Teo, J. T., Swayne, O. B., Cheeran, B., Greenwood, R. J., and Rothwell, J. C. (2011). Human theta burst stimulation enhances subsequent motor learning and increases performance variability. Cereb. Cortex 21, 1627-1638. doi: 10. 1093/cercor/bhq231

Todd, G., Taylor, J. L., and Gandevia, S. C. (2003). Measurement of voluntary activation of fresh and fatigued human muscles using transcranial magnetic stimulation. J. Physiol. 551, 661-671. doi: 10.1113/jphysiol.2003.044099

Todd, G., Taylor, J. L., and Gandevia, S. C. (2004). Reproducible measurement of voluntary activation of human elbow flexors with motor cortical stimulation. J. Appl. Physiol. (1985) 97, 236-242. doi: 10.1152/japplphysiol.01336.2003

Tretriluxana, J., Kantak, S., Tretriluxana, S., Wu, A. D., and Fisher, B. E. (2013). Low frequency repetitive transcranial magnetic stimulation to the non-lesioned hemisphere improves paretic arm reach-to-grasp performance after chronic stroke. Disabil. Rehabil. Assist. Technol. 8, 121-124. doi: 10.3109/17483107. 2012.737136

Ward, N. S., Bestmann, S., Hartwigsen, G., Weiss, M. M., Christensen, L. O., Frackowiak, R. S., et al. (2010). Low-frequency transcranial magnetic stimulation over left dorsal premotor cortex improves the dynamic control of visuospatially cued actions. J. Neurosci. 30, 9216-9223. doi: 10.1523/jneurosci. 4499-09.2010

Williams, P. S., Hoffman, R. L., and Clark, B. C. (2013). Preliminary evidence that anodal transcranial direct current stimulation enhances time to task failure of a sustained submaximal contraction. PLoS One 8:e81418. doi: 10.1371/journal. pone.0081418

Yang, Y. R., Tseng, C. Y., Chiou, S. Y., Liao, K. K., Cheng, S. J., Lai, K. L., et al. (2013). Combination of rTMS and treadmill training modulates corticomotor inhibition and improves walking in Parkinson disease: a randomized trial. Neurorehabil. Neural Repair 27, 79-86. doi: 10.1177/1545968312451915

Ziemann, U. (2004). TMS induced plasticity in human cortex. Rev. Neurosci. 15, 253-266. doi: 10.1515/revneuro.2004.15.4.253

Ziemann, U. (2005). Improving disability in stroke with rTMS. Lancet Neurol. 4, 454-455. doi: 10.1016/s1474-4422(05)70126-5

Conflict of Interest Statement: The authors declare that the research was conducted in the absence of any commercial or financial relationships that could be construed as a potential conflict of interest.

Copyright (C) 2016 Giboin, Thumm, Bertschinger and Gruber. This is an open-access article distributed under the terms of the Creative Commons Attribution License (CC BY). The use, distribution and reproduction in other forums is permitted, provided the original author(s) or licensor are credited and that the original publication in this journal is cited, in accordance with accepted academic practice. No use, distribution or reproduction is permitted which does not comply with these terms. 